\title{
DÜBLIN
}

Technological University Dublin

ARROW@TU Dublin

2003-01-01

\section{Optimization and Comparison of Broadband Monopoles}

\author{
Z. N. Chen \\ Institute for Infocomm Research \\ M.Y. W. Chia \\ Institute for Infocomm Research \\ Max Ammann \\ Technological University Dublin, max.ammann@tudublin.ie
}

Follow this and additional works at: https://arrow.tudublin.ie/engscheceart

Part of the Electrical and Computer Engineering Commons

\section{Recommended Citation}

Chen, Z.N., Chia, M.Y.W \& Ammann, M. (2003) Optimization and Comparison of Broadband Monopoles. IEE Proceedings on Microwaves, Antennas and Propagation, December, 2003, Vol.150, pp. 429-435. doi:10.1049/ip-map:20030856

This Article is brought to you for free and open access by the School of Electrical and Electronic Engineering at ARROW@TU Dublin. It has been accepted for inclusion in Articles by an authorized administrator of ARROW@TU Dublin. For more information, please contact arrow.admin@tudublin.ie, aisling.coyne@tudublin.ie, gerard.connolly@tudublin.ie.

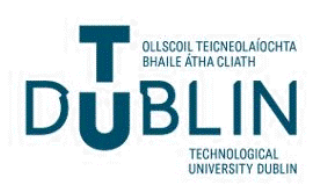




\title{
Optimization and Comparison of Broadband Monopoles
}

\author{
Z.N. Chen, M.Y.W. Chia and M.J. Ammann
}

\begin{abstract}
The impedance and radiation properties of four broadband monopoles are compared, and the parameters of a new roll monopole are optimised experimentally and numerically. The performances of the roll monopole with various numbers of roll turns are discussed and optimised numerically. The measurements and simulations show that, compared with conventional broadband monopoles, the roll monopole features a compact configuration and satisfactory radiation properties across a broad bandwidth.
\end{abstract}

\section{Introduction}

Owing to such attractive merits as simple structure, pure polarisation and omnidirectional radiation, monopoles and their variations have long been applied to a variety of systems. Much effort has been devoted to boost the bandwidth of simple thin-wire monopoles by thickening, loading or folding the wire elements. Conical or skeletal conical, cage, and various loaded monopoles have been proposed [1-4]. However, the major drawback of conical or rotationally symmetric monopoles is their bulky structure. Recently, planar monopoles have been proposed for broadband designs which replace the wire elements with planar elements [5-9]. However, because of the asymmetrical structure, horizontal radiation patterns are not omnidirectional with increased operating frequencies. This degradation more or less mitigates the advantage of the volume reduction. Moreover, for broadband monopoles, the direction of the beam-maximum in the vertical usually varies and the gain of planar monopoles decreases [5-9]. To improve the radiation performances over a broad bandwidth, a sleeve has been used in some designs [10, 11].

In this paper, the impedance and radiation characteristics of a thin-wire monopole, a thick cylindrical monopole, a planar monopole and a roll monopole are examined and compared experimentally and numerically. The broadband roll monopole is essentially evolved from planar monopoles but features a symmetrical structure. Comparisons have shown that, compared with the thick cylindrical monopole and the planar monopole, the roll monopole features not only a broader impedance bandwidth but also more acceptable radiation performance, such as radiation pattern, gain, and beam-maximum direction. Parametric studies of the roll monopole are also implemented numerically.

\footnotetext{
(C) IEE, 2003

IEE Proceedings online no. 20030856

doi:10.1049/ip-map:20030856

Paper first received 6th November 2002 and in revised form 1st July 2003 Online publishing date: 22 October 2003

Z.N. Chen and M.Y.W. Chia are with the Department of Radio Systems, Institute for Infocomm Research, 20 Science Park Road, \#02-34/37 TeleTech Park Singapore 117674

M.J. Ammann is with the School of Electronic and Communications Engineering, Faculty of Engineering, Dublin Institute of Technology, Kevin St., Dublin 8, Ireland
}

\section{Monopoles}

To compare impedance and radiation characteristics, four typical monopoles were designed and investigated. Figure 1 shows the configuration of the monopoles under consideration and a co-ordinate system. All the monopoles are vertically mounted at the centre of a $320 \times 320 \mathrm{~mm}$ ground plane, and a RF signal cable is connected into an SMA connector beneath it. To suppress the additional radiation from the RF feed cable, the cable is enclosed by an absorber layer. The monopoles are excited by a $50 \Omega$ coaxial probe of a $0.6 \mathrm{~mm}$ radius. The bottom edges of the monopoles are parallel to the ground plane with feed gaps $g=1 \mathrm{~mm}$. All the heights of the monopoles are $50 \mathrm{~mm}$.

Monopole 1 and 2 are cylindrical monopoles of diameters $D=2$ and $10 \mathrm{~mm}$, respectively and fed at the centres of their bottoms. Monopole 3 is a planar monopole with a rectangular $0.2 \mathrm{~mm}$ thick copper sheet of width $W=75 \mathrm{~mm}$ and feed point located at $S=30 \mathrm{~mm}$ for the maximum impedance bandwidth. Monopole 4 is formed by uniformly rolling Monopole 3 . The trace of its cross-section can be described by the formula

$$
r=r_{\mathrm{o}}+\alpha \phi
$$

where $r_{\mathrm{o}}$ is the inner radius or minimum radius, $\alpha$ is a constant related to the spacing between two adjacent rolled layers, and denotes the angle ranging from 0 to $360^{\circ} \times \mathrm{N}$, where $N$ is the number of roll turns and may not be an integer. In the measurements, the parameters of the roll are $r_{\mathrm{o}}=4 \mathrm{~mm}, \alpha=0.5 / 360^{\circ}$, and $N=2.5$. The distance between two adjacent layers is $0.5 \mathrm{~mm}$. Detailed parameters of the monopoles are summarised in Table 1.

\section{Comparisons}

Comparisons of the impedance and radiation characteristics of the monopoles were carried out experimentally and numerically. The input impedance was measured using an HP8510C Analyser Network and the radiation patterns were tested in an anechoic chamber using an Orbit Far Field Measurement System. Simulations were implemented using an electromagnetic simulator IE3D software package based on the method of moments. 


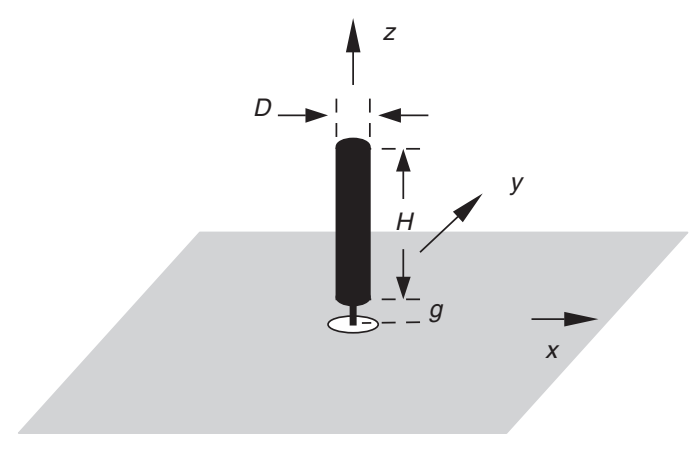

monopole 1,2

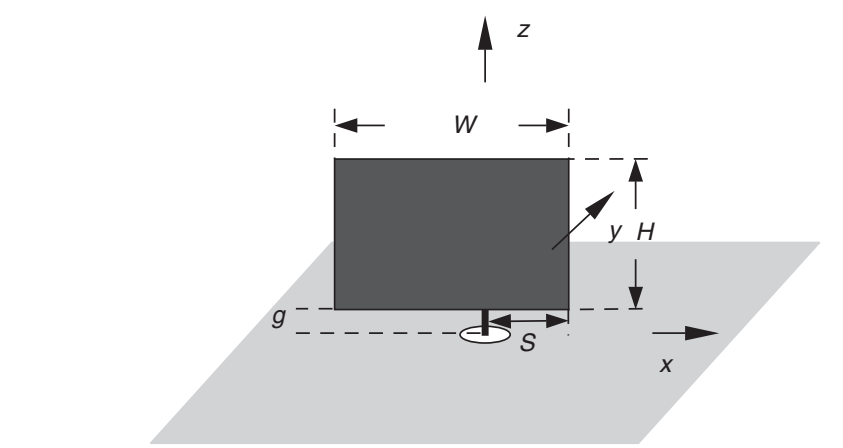

monopole 3

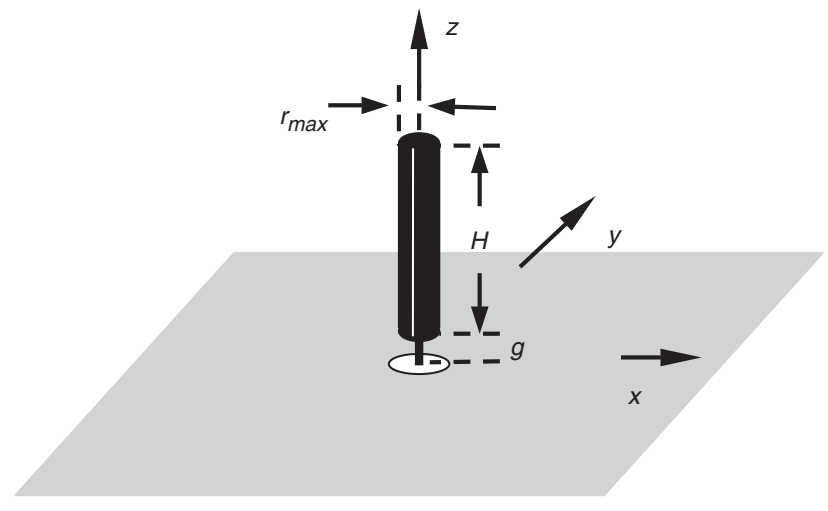

monopole 4

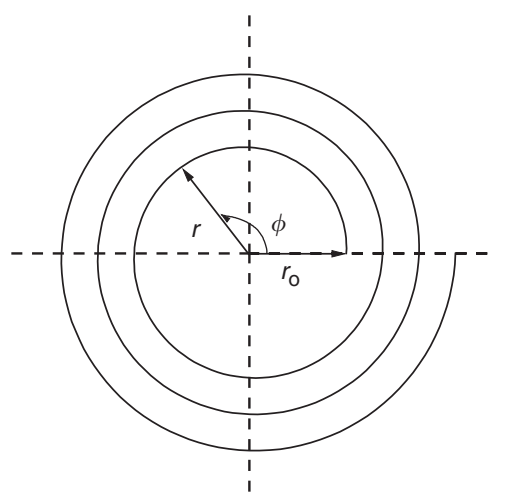

Fig. 1 Geometry of the monopoles under consideration and coordinate system

Table 1: Parameters of monopoles under consideration

\begin{tabular}{lcllll}
\hline Monopole & $W, \mathrm{~mm}$ & $H, \mathrm{~mm}$ & $g, \mathrm{~mm}$ & $S, \mathrm{~mm}$ & $D, \mathrm{~mm}$ \\
\hline 1 & - & 50 & 1 & - & 2 \\
2 & - & 50 & 1 & - & 10 \\
3 & 75 & 50 & 1 & 30 & - \\
4 & - & 50 & 1 & 0 & $r_{\max }=5.5$ \\
\hline
\end{tabular}

\section{Impedance characteristics}

Figure 2 details a comparison of measured and simulated VSWR against frequency. Good agreement between simulation and experiment was obtained. The measured 2:1 VSWR impedance bandwidths detailed in Table 2 show that, as against the $25 \%, 40 \%$ and $53 \%$ bandwidths of monopoles $1-3$, the roll monopole (monopole 4 ) has the largest bandwidth at $71 \%$. Clearly, monopoles 3 and 4 have much broader bandwidths than monopoles 1 and 2 . Furthermore, frequencies for the lower edge of the bandwidths vary from 1.12 to $1.38 \mathrm{GHz}$. It is found that coupling between the layers of the roll leads to parasitic capacitance and the spiral structure introduces parasitic inductance. The parasitic capacitance and inductance result in new resonances so that monopole 4, with a compact size,
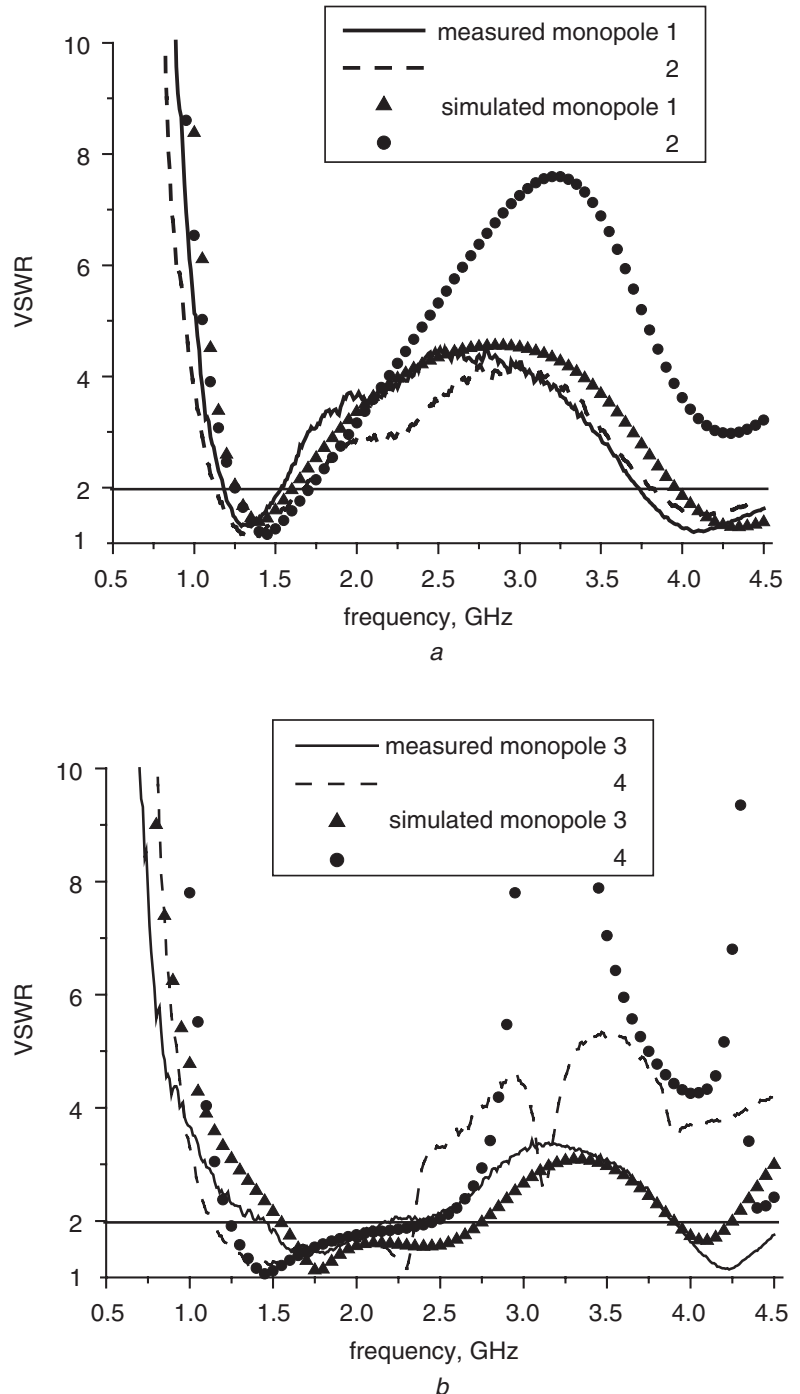

Fig. 2 Measured and simulated VSWR=2:1 against frequency for monopoles $1-4$

Table 2: Measured 2:1 VSWR impedance bandwidths and frequency ranges

\begin{tabular}{llll}
\hline Monopole & $\begin{array}{l}\text { Band- } \\
\text { width }_{2: 1 \text { VswR }}\end{array}$ & $\begin{array}{l}\text { Frequency } \\
\text { range, GHz }\end{array}$ & $\begin{array}{l}\text { Matching } \\
\text { factor }\end{array}$ \\
\hline 1 & $25 \%$ & $1.18 \sim 1.52$ & 1.71 \\
2 & $40 \%$ & $1.12 \sim 1.68$ & 1.50 \\
3 & $53 \%$ & $1.38 \sim 2.38$ & 1.76 \\
4 & $71 \%$ & $1.12 \sim 2.36$ & 1.67 \\
\hline
\end{tabular}

IEE Proc.-Microw. Antennas Propag., Vol. 150, No. 6, December 2003 
realises a broader impedance bandwidth than that of monopole 3.

The measured input impedance of all the monopoles is plotted in Fig. 3. It is seen that monopole 1 has the highest Q-value among the four monopoles. Within a broad frequency range $1.0-3.0 \mathrm{GHz}$, monopoles 3 and 4 have small reactance and resistance varying from 12 to $70 \Omega$. For monopole 4, parasitic resonances with high Q-values have been observed around 2.3 and $3.0 \mathrm{GHz}$ due to the strong electromagnetic coupling between the rolled layers. The phenomenon suggests that the planar spiral structure produces additional inductance and the rolled layers produces additional capacitance.

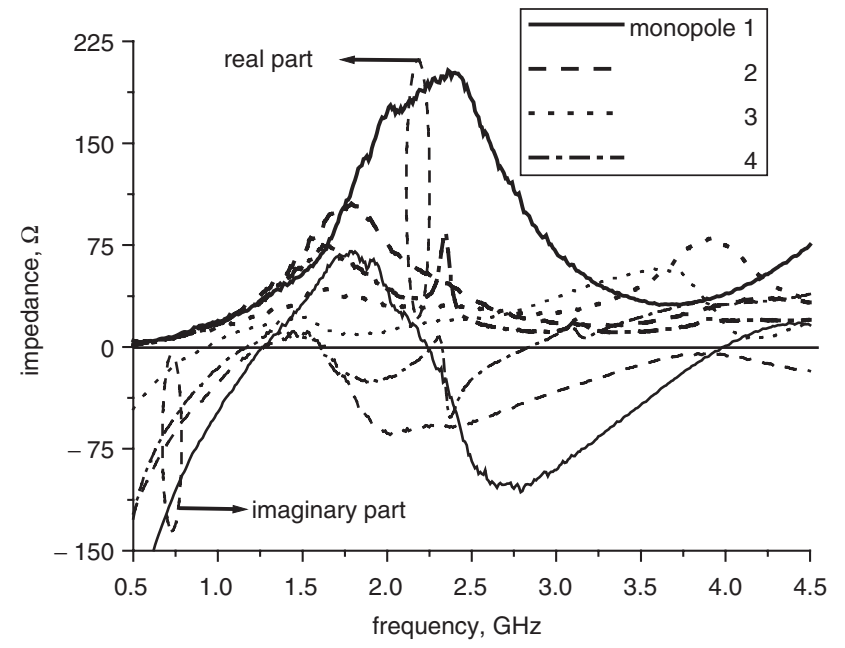

Fig. 3 Comparison of measured input impedance of monopole 1-4

The parameter matching factor $(M F)$ is used to assess the matching performances of broadband monopoles [11]. The $M F$ for VSWR $<2$ is defined as

$$
M F_{\mathrm{VSWR} \leq 2}=\frac{\sum_{m} V S W R_{m} \Delta f_{m}}{B W_{\mathrm{VSWR}} \leq 2}
$$

where $V S W R_{m}$ and $B W_{\mathrm{VSWR}} \leq 2$ are the values of the measured VSWR at the frequency $f_{m}$ and the measured bandwidth for VSWR $\leq 2$, respectively. Thus, there is the following relationship

$$
\sum_{m} \Delta f_{m}=B W_{\mathrm{VSWR} \leq 2}
$$

The calculated $M F$ s are also given in Table 2. Of the monopoles under consideration, monopole 2 with the lowest $M F$ of 1.50 achieved the best matching across the impedance bandwidth. Monopole 4 has an $M F$ of 1.67, which is lower than those of the other two monopoles.

\section{Radiation characteristics}

Measurements of the impedance properties of the monopoles show that the roll monopole has a broad impedance bandwidth, much larger than that of monopole 2 with the same thickness. Therefore, it is important to examine and compare the radiation properties of the four monopoles under investigation. Measurements and simulations are carried out in $x-z, y-z$, and $x-y$ planes and at typical frequencies for each of the monopoles.

For comparison purposes the radiation patterns of monopole 1 were first measured at $1.2,1.4$ and $1.6 \mathrm{GHz}$ as shown in Fig. 4. It is important to notice that the beammaximum directions in the vertical cuts rise from the horizon to $\theta=50 \sim 60^{\circ}$ due to the finite-size ground plane
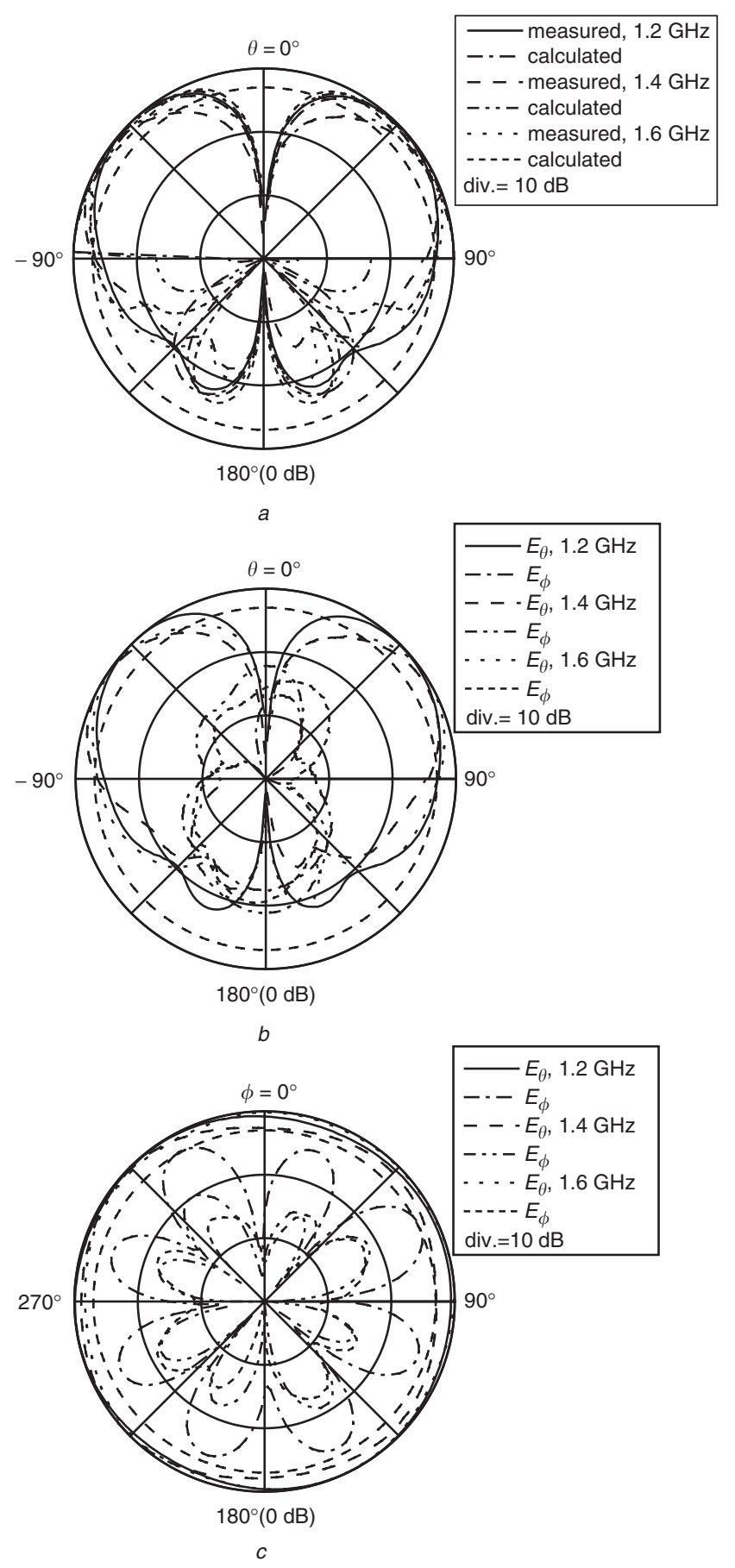

Fig. 4 Measured and simulated radiation patterns of monopole 1 at 1.2, 1.4 and $1.6 \mathrm{GHz}$

a Comparison between the measured and simulated radiation patterns for $E_{\theta}$-components in $x-z$ planes

$b$ Measured radiation patterns for both $E_{\phi^{-}}$and $E_{\theta}$-components in $x-z$ planes

$c$ Measured radiation patterns for both $\mathrm{E}_{\phi^{-}}$and $E_{\theta^{-}}$-components in $x-y$ planes

used in the tests. Within the range $1.2-1.6 \mathrm{GHz}$, the sizes of the ground plane vary from $0.64 \times 0.64 \lambda_{\mathrm{o}}$ to $0.85 \times 0.85 \lambda_{\mathrm{o}}$ and the height of monopole 1 changes from 0.204 to $0.272 \lambda_{\mathrm{o}}$. The calculated and measured radiation patterns for the $E_{\theta}$-components in the $x-z$ planes are compared in Fig. 4a. Calculations are based on the uniform theory of diffraction (UTD) with a thin-wire approximation. The calculated and measured results are in good agreement. Figure $4 b$ suggests that the radiation of the relevant $E_{\phi}$-components is $10 \mathrm{~dB}$ lower than that of the $E_{\theta^{-}}$-component above the ground plane. Figure $4 c$ shows 
the radiation patterns for both $E_{\theta^{-}}$and $E_{\phi^{-}}$-components in the horizontal plane ( $x-y$ plane). The patterns for the $E_{\theta^{-}}$ components are quasi-omnidirectional because of the square ground plane used in the tests and measurement environment. With increase in frequency, the maximum radiation levels of the $E_{\phi}$-components reduce from -2.8 to $-10.9 \mathrm{~dB}$. The symmetry of monopole 1 produces the same radiation patterns in the $y-z$ plane but are not shown for brevity.

The radiation patterns of monopole 2 were then investigated and are shown in Fig. 5. Compared with monopole 1 , monopole 2 with the thicker stem achieves a broader impedance bandwidth of $40 \%$ for $\operatorname{VSWR}<2: 1$, but almost the same radiation performance within the bandwidth. This suggests that the monopole should be kept structurally symmetrical to obtain stable radiation performances within a broad bandwidth.

However, Fig. 6 shows that the planar monopole (Monopole 3) with asymmetrical geometry, which is usually used to increase the bandwidth of monopoles of reduced size, suffers from degradation of the radiation patterns. The test frequency range 1.0 to $2.4 \mathrm{GHz}$ is much higher than that for monopole 1 or 2 , although the patterns are measured only at $1.4,1.8$ and $2.2 \mathrm{GHz}$. Figure $6 a$ demonstrates that, in the $x-z$ planes, the $E_{\phi}$-component levels are still low but the radiation of $E_{\theta}$-components features high levels and end-fire patterns. The asymmetry of the patterns ( $x-z$ planes) for the $E_{\theta}$-components is caused
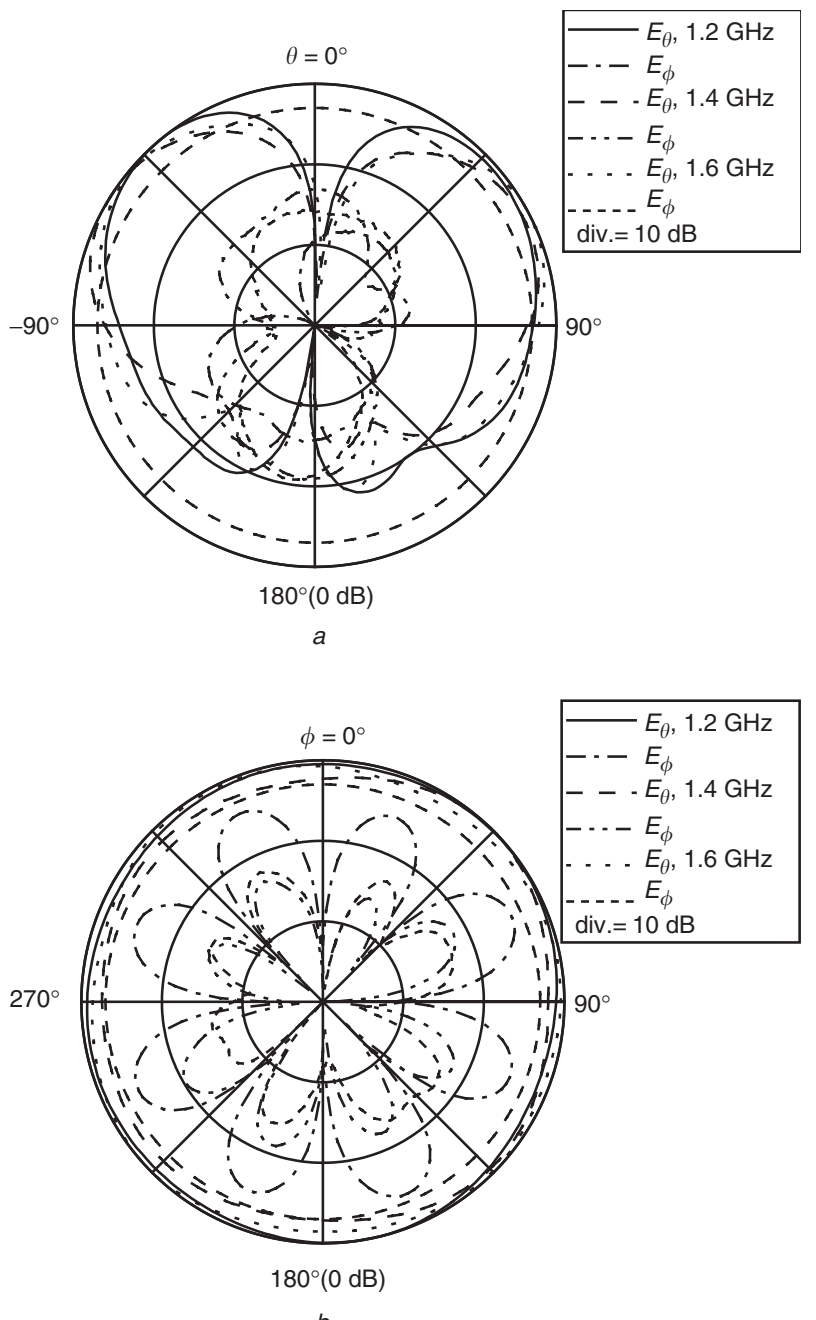

Fig. 5 Measured radiation patterns for both $E_{\phi^{-}}$and $E_{\theta^{-}}$ components of Monopole 2 at 1.2, 1.4 and $1.6 \mathrm{GHz}$

$a$ In $x-z$ planes

$b$ In $x-y$ planes mainly by the asymmetrical feed configuration. Figure $6 b$ shows that in the $y-z$ planes, as against the end-fire patterns for the $E_{\phi}$-components, the patterns for the $E_{\theta}$-components are typical broadside. However, the $E_{\phi}$-component levels are almost the same as the $E_{\theta}$-components because both $x$ and $z$-components of the electric currents are excited at the radiating sheet simultaneously. Also, the severe asymmetry of the monopole causes degradation of the omnidirectional radiation patterns of the $E_{\theta}$-components in the horizontal plane $(x-y$ plane), as shown in Fig. $6 c$. This degradation becomes worse when the operating frequency increases.

Finally, monopole 4, which was proposed to alleviate the degraded radiation performance of the broadband planar monopole, was studied. The radiation patterns were also measured within the frequency range $1.0-2.4 \mathrm{GHz}$ although for comparison purposes, only the patterns measured at 1.4, 1.8 and $2.2 \mathrm{GHz}$ are shown here. Compared with the radiation patterns shown in Figs. $4 b$ and $5 a$, Figs. $7 a$ and $7 b$ demonstrate the same radiation properties above the ground plane and slightly lower back radiation levels. Figure $7 c$ shows that, because of the symmetrical-like structure, the radiation patterns for both $E_{\phi^{-}}$and $E_{\theta^{-}}$ components are almost omnidirectional when considering the impact of the ground plane on the radiation performances observed in the measurements on monopoles 1,2 or 3 .

\section{Comparison and discussion}

Comparisons of the radiation properties of the four monopoles were made in detail.. The gains are shown in Fig. 8, where gains for monopoles 3 and 4 are measured in both $x-z$ and $y-z$ planes. The comparison shows that within the operating bandwidths, monopole 4 has achieved quite stable and high gains ranging from 3.2 to $4.6 \mathrm{dBi}$ in both planes. Monopole 3 suffers from severe variation of the gain at different frequencies. It is seen that the gains vary from 2.3 to $5.6 \mathrm{dBi}$ in the $x-z$ planes and from 2.3 to $4.8 \mathrm{dBi}$ in the $y-z$ planes. The simulated gain for monopole 4 is done above the ground plane and is about $0.5 \mathrm{dBi}$ higher than the measured gain due to the infinite ground plane used in simulations.

Figure 9 shows that the beam-maximum directions for monopoles 1, 2 and 4 change in the same way over the frequency range $1.4-2.2 \mathrm{GHz}$; between $56^{\circ}$ and $63^{\circ}$, except for $45^{\circ}$ at $1.2 \mathrm{GHz}$. However, the beam-maximum directions of monopole 3 vary from $14^{\circ}$ (at $1.6 \mathrm{GHz}$ ) to $60^{\circ}$ (at $2.0 \mathrm{GHz}$ ) in the $y-z$ plane.

To assess the radiation properties of the monopoles in the horizontal plane, Fig. 10 demonstrates the dip depths of the radiation patterns for the $E_{\theta}$-components. The dip depth is used to measure the variation from a purely omnidirectional pattern. Considering the $1-2 \mathrm{~dB}$ tolerance of the testing environment, due to the finite-size ground plane and RF signal cable, monopole 4 has an omnidirectional performance with dip depths less than $-3.4 \mathrm{~dB}$ across the bandwidth, which is nearly the same as that of monopoles 1 and 2 . In contrast, within the bandwidth, monopole 3 dip depths vary from -5.1 to $-11.3 \mathrm{~dB}$. Therefore, the roll monopole has almost the same radiation properties as the cylindrical monopoles but a much broader impedance bandwidth, similar to that of a planar monopole.

\section{Optimisation}

Comparisons have shown that the roll monopole has the advantages of broad impedance bandwidth and satisfactory radiation performance over cylindrical and planar monopoles. Therefore, the impedance characteristics of roll 

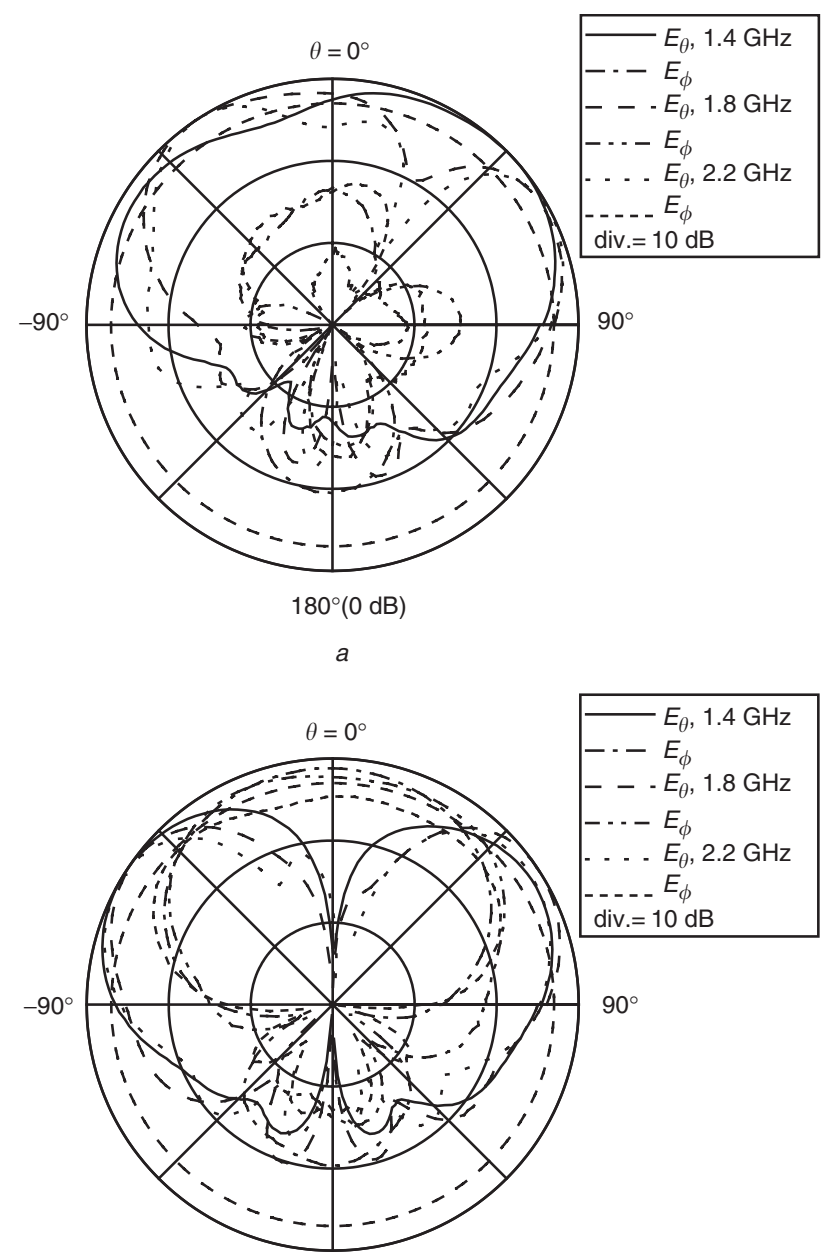

$180^{\circ}(0 \mathrm{~dB})$

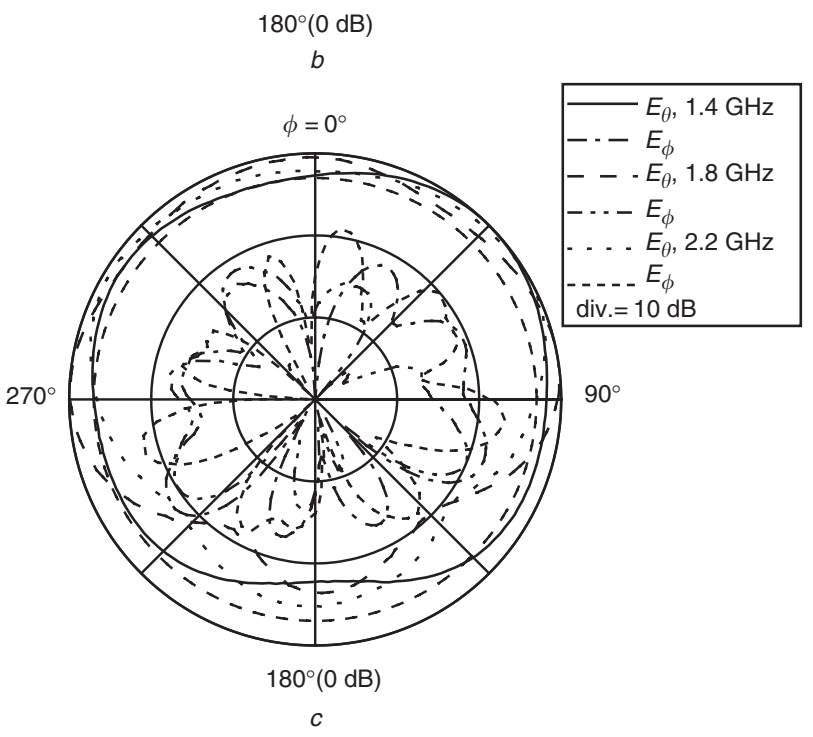

Fig. 6 Measured radiation patterns for both $E_{\phi^{-}}$and $E_{\theta^{-}}$ components of monopole 3 at 1.4, 1.8 and $2.2 \mathrm{GHz}$

$a$ In $x-z$ planes

$b$ In $y-z$ planes

$c$ In $x-y$ planes

monopoles with different parameters, such as the numbers of roll turns $N$ and feed gap $g$, were examined numerically.

The roll monopoles have the same parameters as monopole 4 with $g=1 \mathrm{~mm}$, but $N$ varies from 1 to 3 . Figure 11 shows the simulated VSWR. The case with $N=1$ achieves the widest bandwidth of $78 \%(1.22-2.78 \mathrm{GHz})$ for VSWR $<2: 1$, although the impedance matching is not good owing to the VSWR close to 2:1. By making a trade-off
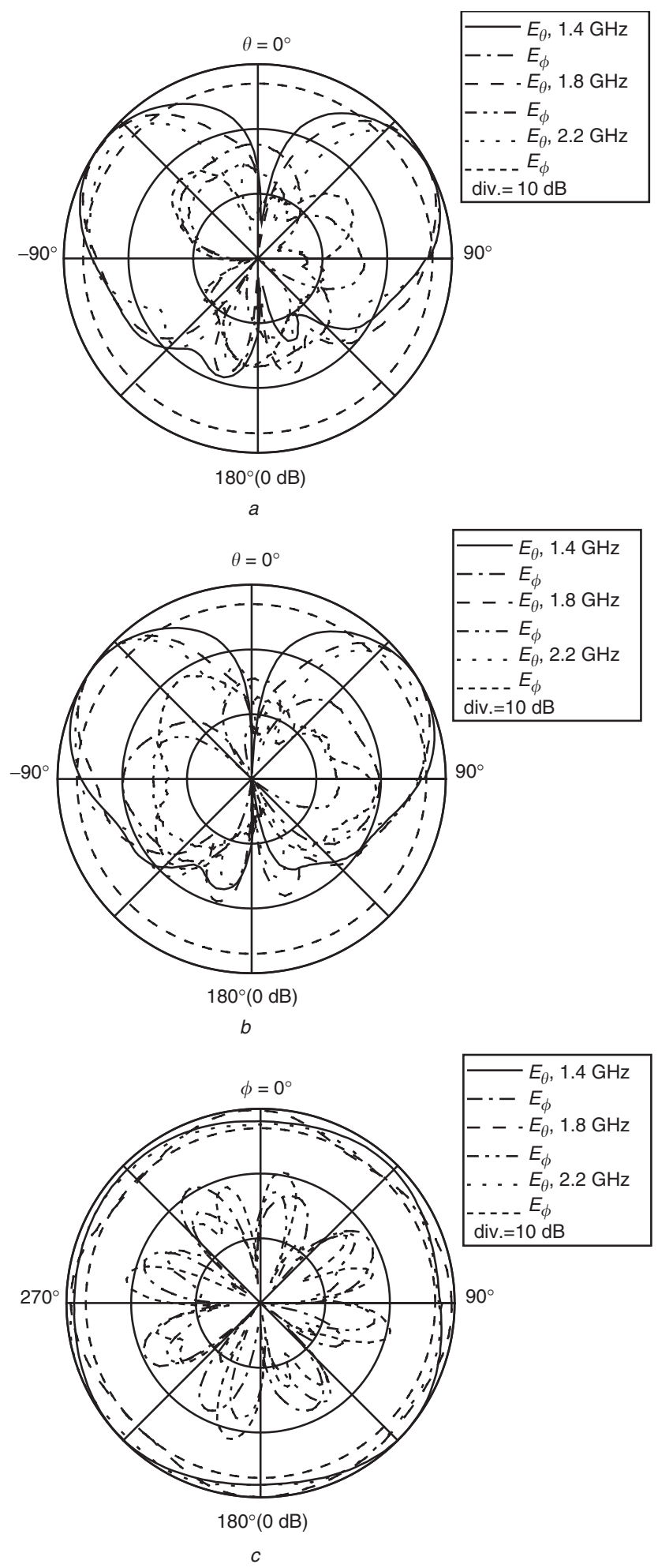

Fig. 7 Measured radiation patterns for both $E_{\phi^{-}}$and $E_{\theta^{-}}$ components of monopole 4 at $1.4,1.8$ and $2.2 \mathrm{GHz}$

$a$ In $x-z$ planes

$b$ In $y-z$ planes

$c$ In $x-y$ planes

between bandwidth and matching condition, the case with $N=2.5$ was selected and manufactured for tests, giving a simulated bandwidth of $67 \% \quad(1.23-2.48 \mathrm{GHz})$ for VSWR $<2: 1$. Furthermore, parasitic resonances occurred in the cases $N=1.5$ and 3 because of the introduction of additional reactance between the rolled layers. Thus, both cases, $N=1$ and 2.5, are good options for broad applications. 


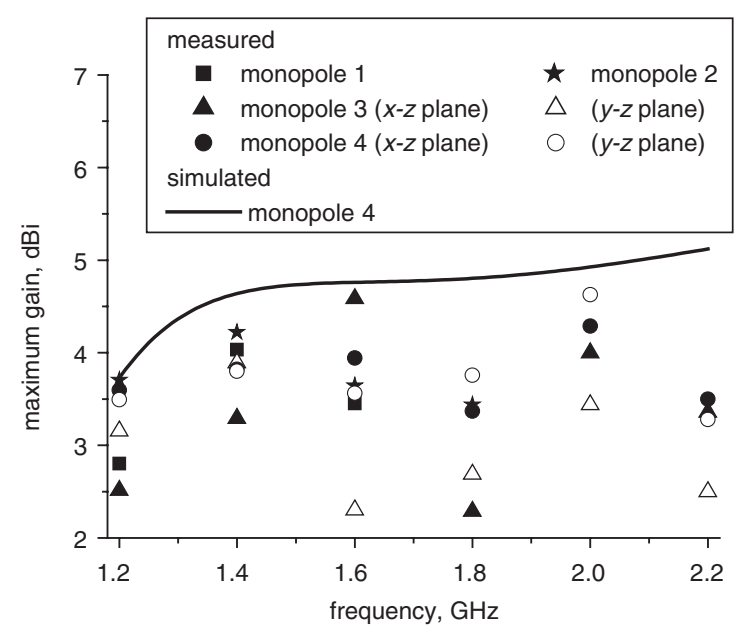

Fig. 8 Comparison of measured and simulated maximum gains of monopoles 1-4

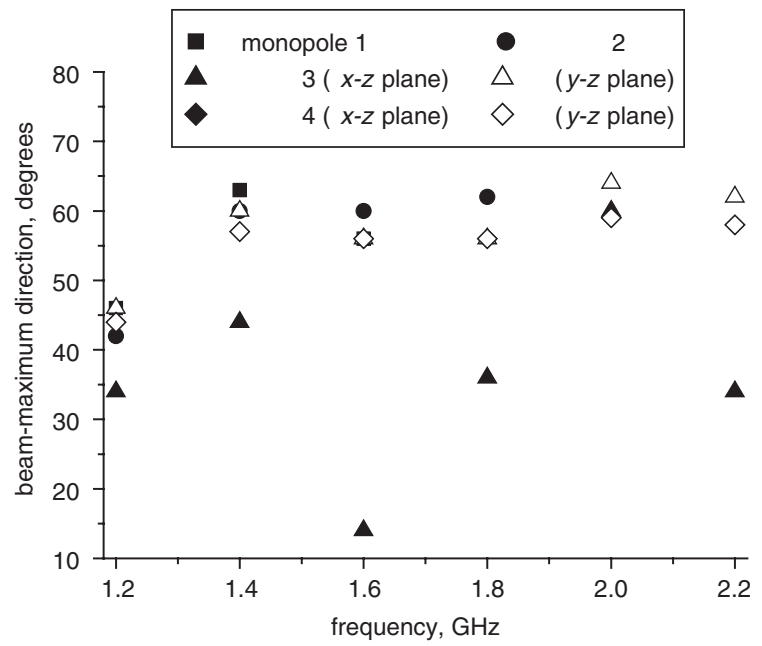

Fig. 9 Comparison of measured beam-maximum direction of monopoles 1-4

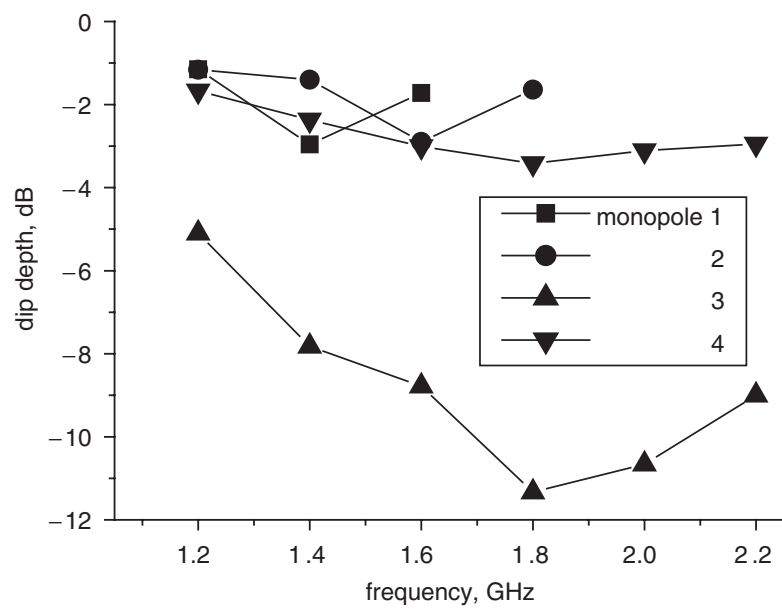

Fig. 10 Comparison of measured dip depths of radiation patterns for $E_{\theta}$-components in the $x-y$ planes of monopoles $1-4$

Parametric studies of roll monopoles with $N=1,2,2.5$ and 3 , and varying feed gaps $g=1,2$, and $3 \mathrm{~mm}$ were carried out. For brevity, Fig. 12 shows only comparisons of the VSWR of the roll monopole with $N=2.5$. The feed

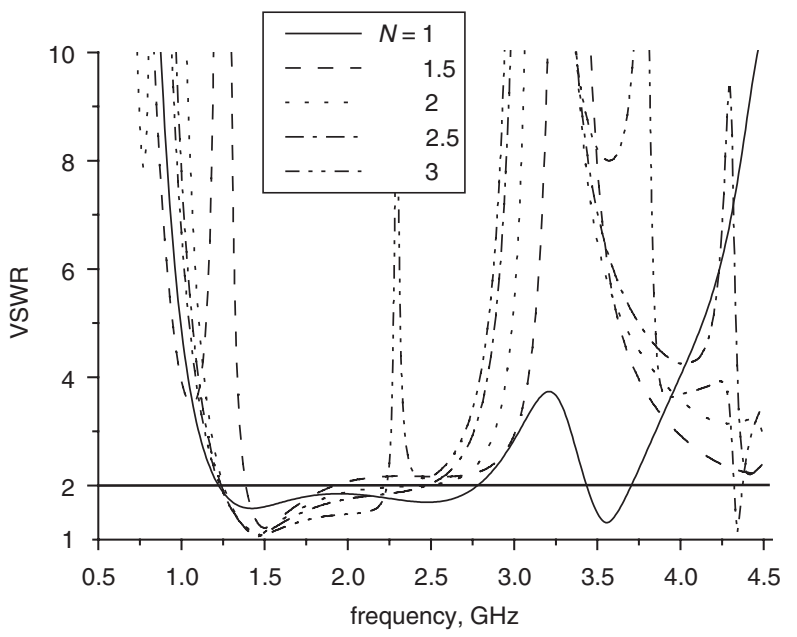

Fig. 11 Comparison of simulated VSWR of roll monopole for the varying number of roll turns

point is located at the point $\left(r=4 \mathrm{~mm}, \phi=0^{\circ}\right)$. The 2:1 VSWR bandwidth of $84 \%$ was realised with a feed gap $g=3 \mathrm{~mm}$. The bandwidth decreased for feed gap greater than $3 \mathrm{~mm}$.

Figure $13 a$ and $b$ compare the VSWR of roll monopoles with different feed point locations. For cases with $g=1$ and $3 \mathrm{~mm}$, the broadest bandwidths are $67 \%$ and $84 \%$, respectively. The optimal location for the broadest bandwidth is the point $\left(r=4 \mathrm{~mm}, \phi=0^{\circ}\right)$. For the other locations, the bandwidths are about $50 \%$ for $g=1 \mathrm{~mm}$ and $30 \%$ for $g=3 \mathrm{~mm}$.

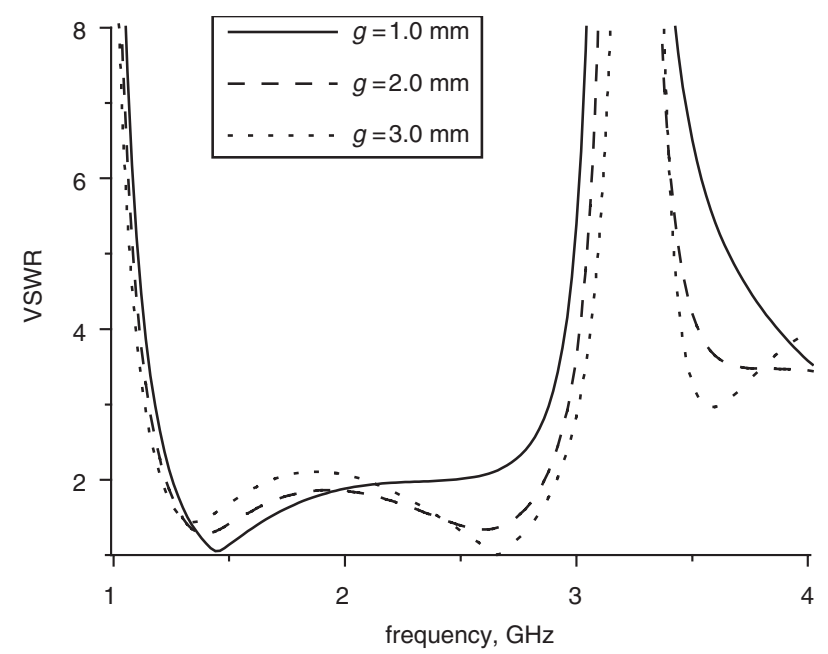

Fig. 12 Comparison of simulated VSWR of roll monopole with $N=2.5$ for different feed gaps

The number of roll turns and feed gap width affect the bandwidth. The optimised location of the feed point is at the inner start point.

\section{Conclusions}

Comparisons of the impedance and radiation characteristics of four monopoles have been carried out experimentally and numerically. A roll monopole antenna has been presented for broadband applications and its parameters have been optimised numerically. Studies have shown that 


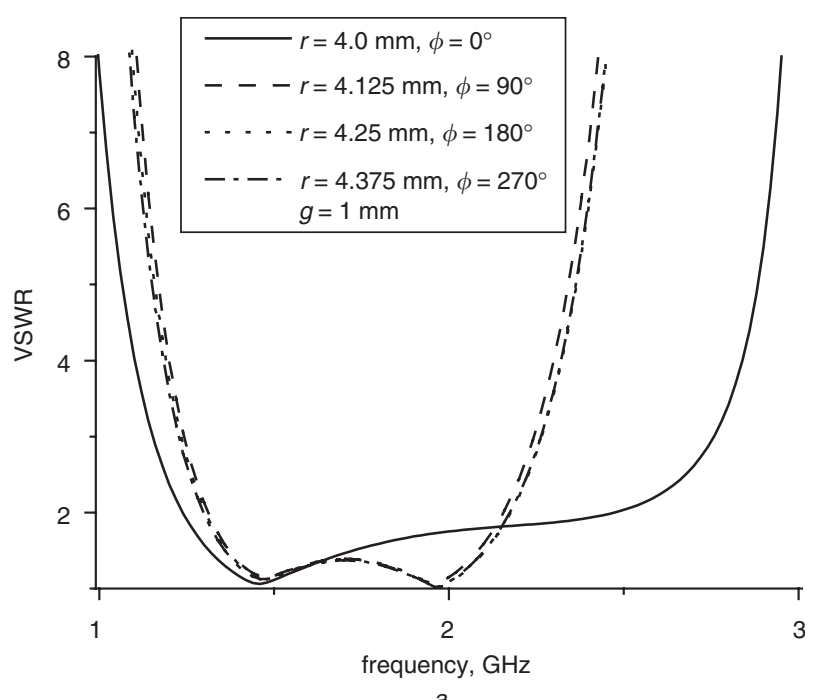

a

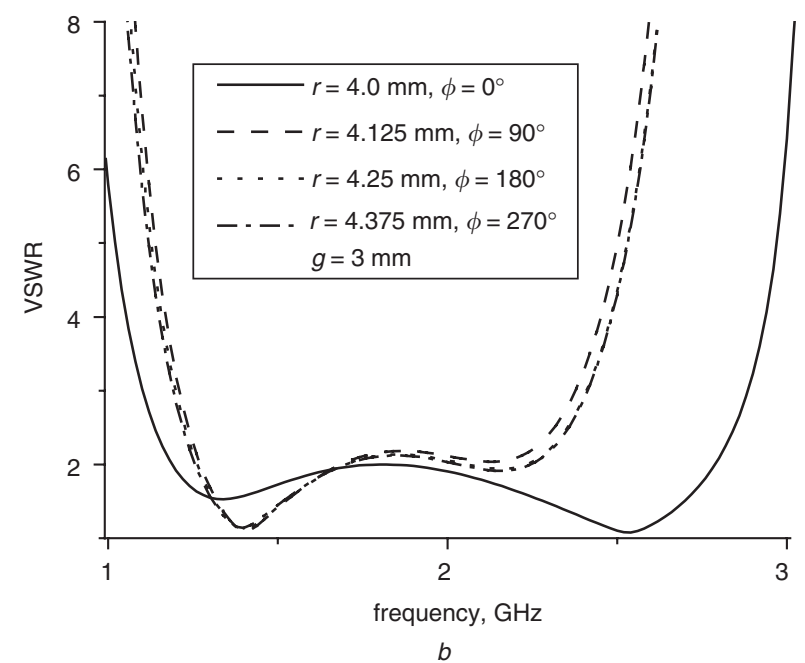

Fig. 13 Comparison of simulated VSWR of roll monopole for different feed point locations

a $g=1 \mathrm{~mm}$,

$b g=3 \mathrm{~mm}$ by combining the advantages of the broad impedance bandwidth of a planar monopole and the stable radiation properties of a cylindrical monopole, the roll monopole features a compact structure with remarkably broad bandwidths for both impedance and radiation characteristics.

\section{References}

1 Kawakami, H., and Sato, G.: 'Broadband characteristics of rotationally symmetric antennas and thin wire constructs', IEEE Trans. Antennas Propag., 1987, 35, pp. 26-32

2 Nakano, H., Ikeda, N., Wu, Y., Suzuki, R., Mimaki, H., and Yamauchi, J.: 'Realization of dual-frequency and wide-band VSWR performances using normal helical and inverted-F antennas', IEEE Trans. Antennas Propag., 1998, 46, pp. 788-793

3 Rogers, S.D., and Butler, C.M.: "Cage antennas optimised for bandwidth', Electron. Lett., 2000, 36, (11), pp. 932-933

4 Cho, W., Kanda, M., Hwang, H., and Howard, M.W.: 'A disk-loaded thick cylindrical dipole antenna for validation of an EMC test site from 30 to $300 \mathrm{MHz}$, IEEE Trans. Electromagn. Compat., 2000, 42, pp. $172-180$

5 Brown, G.H., and Woodward, O.M.: 'Experimentally determined radiation characteristics of conical and triangular antennas', $R C A$ Rev., 1952, 13, (4), pp. 425-452

6 Honda, S., Ito, M., Seki, H., and Jinbo, Y.: 'A disk monopole antenna with 1:8 impedance bandwidth and omnidirectional radiation pattern'. Proc. ISAP92, Sapporo, Japan, 1992, pp. 1145-1148

7 Agrawall, N.P., Kumar, G., and Ray, K.P.: 'Wide-band planar monopole antenna', IEEE Trans. Antennas Propag., 1998, 46, pp. 294-295

8 Ammann, M.J.: 'Square planar monopole antenna'. Presented at National Conf. Antennas \& Propag., York, England, 1999, pp. 37-40

9 Chen, Z.N.: 'Impedance characteristics of planar bow-tie-like monopole antennas', Electron. Lett., 2000, 36, (13), pp. 1100-1101

10 King, R.W.P.: 'Asymmetric driven antenna and the sleeve dipole', Proc. IRE, 1950, 38, pp. 1154-1164

11 Chen, Z.N., Hirasawa, K., and Wu, K.: 'A novel top-sleeve monopole in two parallel plates', IEEE Trans. Antennas Propag., 2001, 49, pp. $438-443$ 